\title{
Dimension, entropy and Lyapunov exponents
}

\author{
LAI-SANG YOUNG \\ Department of Mathematics, Michigan State University, East Lansing, MI 48824
}

(Received 8 April 1982)

Abstract. We consider diffeomorphisms of surfaces leaving invariant an ergodic Borel probability measure $\mu$. Define $\operatorname{HD}(\mu)$ to be the infimum of Hausdorff dimension of sets having full $\mu$-measure. We prove a formula relating $\operatorname{HD}(\mu)$ to the entropy and Lyapunov exponents of the map. Other classical notions of fractional dimension such as capacity and Rényi dimension are discussed. They are shown to be equal to Hausdorff dimension in the present context.

Hausdorff dimension was introduced in 1919. It is a notion of size, useful for distinguishing between sets of Lebesgue measure zero. This is particularly relevant in the study of non-conservative dynamical systems where many interesting invariant sets are null in the sense of Lebesgue. Entropy was defined by Kolmogorov in the 1950's [11]. If $f$ is a transformation preserving a probability measure $\mu$, then the $\mu$-entropy of $f$, denoted $h_{\mu}(f)$, measures the asymptotic growth in information through iterating $f$. In the context of smooth dynamical systems, $h_{\mu}(f)$ is related to the rate at which points are being dispersed. The concept of Lyapunov exponents, as we use the term here, is due to Oseledec and came later in the 60's [16]. If $f$ is a differentiable map of a manifold into itself, these numbers describe the asymptotic behaviour of the derivative of $f$. When $f$ is smooth enough, they dominate the local behaviour of the system.

All three of these invariants have been studied a great deal. Connections between entropy and dimension for specific examples have been observed long ago by many people. (See, for instance, Billingsley's book [2].) The inequality of Margulis and Ruelle [19] and the formula of Pesin [17] highlight the connection between entropy and Lyapunov exponents. More recently, the work of Douady and Oesterlé [6] and the conjectures of Yorke [8], [9] led to a search for the relations between the dimension of attractors and the exponents of the map.

In this paper we show that a natural relation exists between these three invariants if entropy and Hausdorff. dimension are looked at from a certain perspective. This relation holds very generally for all diffeomorphisms of surfaces and could perhaps be extended to mappings of higher dimensional manifolds. If $\mu$ is a probability measure on a metric space, let $\operatorname{HD}(\mu)$ denote the Hausdorff dimension of the 'smallest' set of full measure. We state our main result here, leaving precise definitions for later. 
MAIN THEOREM. Let $f: M \circlearrowleft$ be a $C^{2}$ diffeomorphism of a compact surface $M$ and let $\mu$ be an ergodic Borel probability measure with exponents $\lambda_{1} \geq \lambda_{2}$. Then

$$
\mathrm{HD}(\mu)=h_{\mu}(f)\left[\frac{1}{\lambda_{1}}-\frac{1}{\lambda_{2}}\right]
$$

whenever the right side of this equation is not $0 / 0$.

In $\S 1$ we motivate this formula with some examples. $\S 2$ is devoted to background material and $\S 3$ to the proof of the main result. In $\S 4$ we discuss other notions of dimension such as capacity and Rényi dimension and show their equivalence under $\vec{r}$ the same hypotheses. $\$ 5$ consists of corollaries and remarks.

This work was partially inspired by the papers of Manning [14] and Mañe [13]. I am grateful to F. Ledrappier and J. Yorke for constantly sharing their insight with me. Thanks are also due to A. Katok for helpful conversations.

\section{Examples}

Throughout the paper we use $\operatorname{HD}(X)$ to denote the Hausdorff dimension of the set $X$.

\section{A. Dyadic expansions}

For $x \in[0,1]$, let $x=0 \cdot x_{1} x_{2} x_{3} \cdots$ be the dyadic expansion of $x$. Fix $0<p<1$ and let

$$
X=\left\{x \in[0,1]: \lim _{n \rightarrow \infty} \frac{1}{n} \sum_{i=0}^{n-1} x_{i}=p\right\} .
$$

Besicovitch [1] and Eggleston [7] proved several decades ago that

$$
H D(X)=\frac{1}{\log 2}[-p \log p-(1-p) \log (1-p)] \text {. }
$$

We rephrase this result slightly. Let $f:[0,1] \leftrightharpoons$ be given by $f x=2 x \bmod 1$ and let $\mu$ be the unique ( $f$-invariant) Bernoulli probability measure on $[0,1]$ satisfying

$$
\mu\left\{x \in[0,1]: x_{1}=1\right\}=p .
$$

Then the $\mu$-Lyapunov exponent of $f$, which we denote by $\lambda$, is $\log f^{\prime}=\log 2$ and the result of Besicovitch and Eggleston says that there is a set $X \subset[0,1]$ with $\mu X=1$ and

$$
\mathrm{HD}(X)=\frac{h_{\mu}(f)}{\lambda}
$$

\section{B. Linear horseshoes}

Recall that if $C=\bigcap_{n=1}^{\infty} C_{n}$ is the standard Cantor set with $C_{n}$ consisting of $p^{n}$ subintervals of length $(1 / q)^{n}$ each, then

$$
\mathrm{HD}(C)=\frac{\log p}{\log q}
$$

Consider maps of the form shown in figure 1. That is, a square $R$ is stretched and contracted linearly, bent around finitely many times and then superimposed 


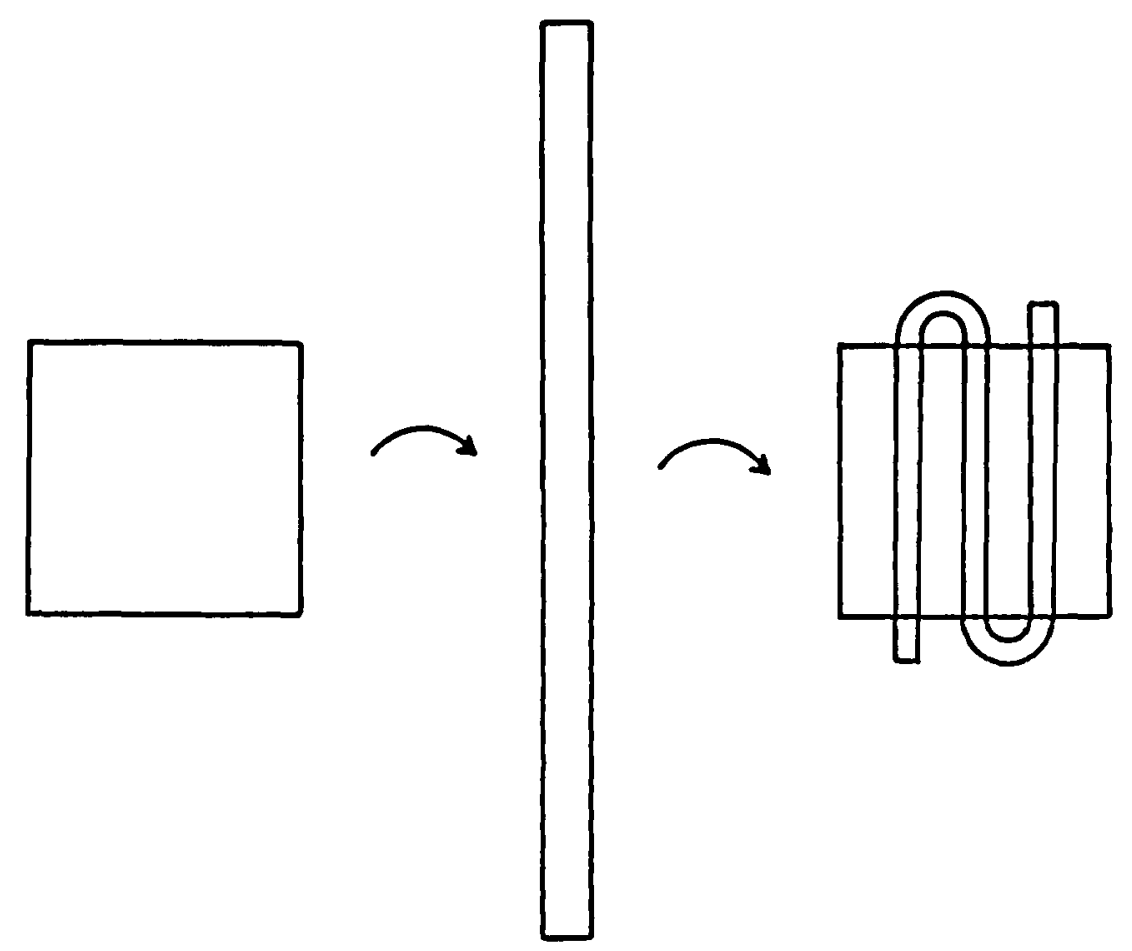

FIGURE 1

on itself as shown. Call this map $f$ and let $\Lambda=\bigcap_{n \in Z} f^{n} R$. We have

$$
D f \mid \Lambda=\left(\begin{array}{cc} 
\pm D f^{s} & 0 \\
0 & \pm D f^{u}
\end{array}\right) .
$$

A precise treatment of horseshoes is given in [15].

Let $L_{H}$ be a horizontal line through $\Lambda$. Then

$$
\operatorname{HD}\left(L_{\mathbf{H}} \cap \Lambda\right)=\frac{h(f)}{\log \left|D f^{s}\right|^{-1}}
$$

where $h(f)=\log$ (\# of crossings) is the topological entropy of $f$. Similarly if $L_{\mathrm{V}}$ is a vertical line through $\Lambda$, then

$$
\operatorname{HD}\left(L_{v} \cap \Lambda\right)=\frac{h(f)}{\log \left|D f^{u}\right|}
$$

In this linear case Hausdorff dimension adds and we have

$$
\operatorname{HD}(\Lambda)=h(f)\left[\frac{1}{\log \left|D f^{u}\right|}-\frac{1}{\log \left|D f^{s}\right|}\right] \text {. }
$$

C. Anosov toral automorphisms

Let $L: \mathbb{R}^{2} \circlearrowleft$ be a linear map with integer coefficients and real eigenvalues $\tau$ and $1 / \tau, \tau>1$, and let $\dot{L}: T^{2} \leftrightarrows$ be the induced automorphism of $T^{2}$. Then $\dot{L}$ preserves Haar measure $\mu$ and $h_{\mu}(\tilde{L})=\log \tau$ so that

$$
h_{\mu}(\check{L})\left[\frac{1}{\log \tau}-\frac{1}{\log \tau^{-1}}\right]=2=\mathrm{HD}(\mu)
$$


2. Basic facts and known results

In this section we collect some facts that will be used in the proof of the main theorem. Notations adopted here are used throughout.

\section{A. Hausdorff dimension}

Let $X$ be a metric space. For $x \in X$ and $\rho>0$, let

$$
B_{\rho}(x)=\{y: d(x, y) \leq \rho\} .
$$

The diameter of a cover $\kappa$ of $X=\sup \{\operatorname{diam} A: A \in \kappa\}$. Then

$$
\operatorname{HD}(X)=\inf \left\{\alpha: \lim _{\boldsymbol{\alpha} \rightarrow 0} \inf _{\substack{\alpha-\text { cover of } X \\ \text { diam } \kappa \leq \varepsilon}} \sum_{A \in \kappa}(\operatorname{diam} A)^{\alpha}=0\right\} .
$$

Since our measures live on manifolds, we may assume via $C^{1}$ charts that we are working in $\mathbb{R}^{n}$. For the rest of $\S 2 \mathrm{~A}$, let $\mu$ be a finite non-atomic Borel measure on $\mathbb{R}^{n}$.

We take a local or pointwise approach to Hausdorff dimension. The following proposition is essentially borrowed from [2], modified slightly to suit our purposes.

Proposition 2.1. Let $\Lambda \subset \mathbb{R}^{n}$ be measurable and have $\mu \Lambda>0$. Suppose that for every $x \in \Lambda$,

$$
\underline{\delta} \leq \liminf _{\rho \rightarrow 0} \frac{\log \mu B_{\rho}(x)}{\log \rho} \leq \limsup \frac{\log \mu B_{\rho}(x)}{\log \rho} \leq \bar{\delta} .
$$

Then

$$
\underline{\delta} \leq \mathrm{HD}(\Lambda) \leq \bar{\delta} .
$$

Remark. All limits remain unchanged if the continuous variable $\rho$ is replaced by any sequence $\left\{\rho_{n}\right\}$ with $\rho_{n} \downarrow 0$ and $\log \rho_{n+1} / \log \rho_{n} \rightarrow 1$.

Proof. For $U \subset \mathbb{R}^{n}$, let $\mathscr{R}(U, \varepsilon)=\left\{B_{\rho}(x): x \in U, \rho \leq \varepsilon\right\}$ and define

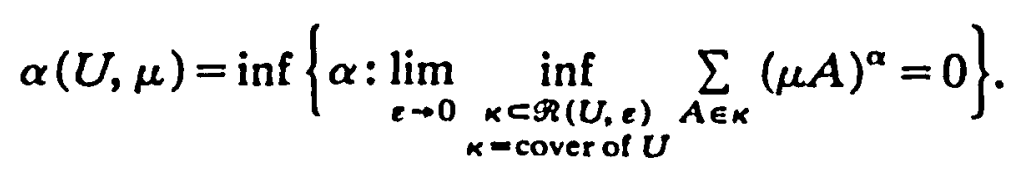

If $U$ is measurable and has positive $\mu$-measure, then it is easy to verify that $\alpha(U, \mu)=1$.

Fix $U \subset \mathbb{R}^{n}$ with $\mu U>0$. We first prove the proposition under the stronger assumption that $\exists \varepsilon_{0}>0$ s.t. $\forall x \in U$ and $\forall \rho \leq \varepsilon_{0}$,

$$
\delta \leq \log \mu B_{\rho}(x) / \log \rho \leq \bar{\delta} \text {. }
$$

Let $\alpha>1$. Let $0<\varepsilon_{1}<\varepsilon_{0}$ and $\varepsilon_{2}>0$ be given. Since $\alpha(U, \mu)=1, \exists$ a cover $\kappa \subset$ $\mathscr{R}\left(U, \varepsilon_{1}\right)$ of $U$ satisfying

$$
\sum_{A \in \kappa}(\mu A)^{\alpha} \leq \varepsilon_{2}
$$

Our hypothesis here is that for $A \in \mathscr{R}\left(U, \varepsilon_{0}\right)$,

$$
(\operatorname{diam} A)^{\bar{\delta}} \leq 2^{\bar{\delta}} \mu A \text {. }
$$


Therefore

$$
\sum_{A \in \kappa}(\operatorname{diam} A)^{\alpha \bar{\delta}}<2^{\alpha \bar{\delta}} \varepsilon_{2}
$$

and hence $\operatorname{HD}(U) \leq \alpha \bar{\delta}$. As for the other inequality note that the definition of Hausdorff dimension remains unchanged if we restrict ourselves to subcovers of $\mathscr{R}(U, \varepsilon)$. We have

$$
\sum_{A \in \kappa}(\operatorname{diam} A)^{\delta} \geq 2^{\delta} \sum_{A \in \kappa} \mu A \geq 2^{\delta} \mu U
$$

for every cover $\kappa \subset \mathscr{R}\left(U, \varepsilon_{0}\right)$ of $U$, which proves $\operatorname{HD}(U) \geq \underline{\delta}$.

To complete the proof of the proposition we fix $\varepsilon>0$ and an acceptable sequence $\rho_{n} \downarrow 0$. Let

$$
\Lambda_{k}=\left\{x \in \Lambda: \forall n \geq k, \underline{\delta}-\varepsilon \leq \log \mu B_{\rho_{n}}(x) / \log \rho_{n} \leq \bar{\delta}+\varepsilon\right\} .
$$

Since $x \mapsto \mu B_{\rho}(x)$ is a measurable function, the sets $\Lambda_{k}$ are measurable and $\mu \Lambda_{k} \uparrow \mu \Lambda$. The argument above applies to each $\Lambda_{k}$. Let $k \uparrow \infty$ and then $\varepsilon \downarrow 0$.

\section{B. Entropy}

As in the case of Hausdorff dimension we take a local approach to entropy. Let $f: M \circlearrowleft$ be a diffeomorphism of a compact Riemannian manifold, let $\mu$ be an ergodic Borel probability measure and let $\phi: M \rightarrow \mathbb{R}^{+}$be a function on $M$. For $x \in M$ and $n_{1}, n_{2} \in \mathbb{Z}^{+}$, define

$$
V\left(x, \phi, n_{1}, n_{2}\right)=\left\{y \in M: d\left(f^{k} x, f^{k} y\right) \leq \phi\left(f^{k} x\right),-n_{2} \leq k \leq n_{1}\right\} .
$$

We write $V\left(x, \varepsilon, n_{1}, n_{2}\right)$ for $V\left(x, \phi, n_{1}, n_{2}\right)$ when $\phi \equiv \varepsilon$.

Here are the two estimates that will be used:

Theorem (Mañé [13]). If $\int-\log \phi d \mu<\infty$, then for $\mu$-a.e. $x$,

$$
\limsup _{n_{1}, n_{2} \rightarrow \infty} \frac{-1}{n_{1}+n_{2}} \log \mu V\left(x, \phi, n_{1}, n_{2}\right) \leq h_{\mu}(f) \text {. }
$$

THEOREM (Brin-Katok [5]). For $\mu$-a.e. $x$,

$$
\lim _{\varepsilon \rightarrow 0} \liminf _{n_{1}, n_{2} \rightarrow \infty} \frac{-1}{n_{1}+n_{2}} \log \mu V\left(x, \varepsilon, n_{1}, n_{2}\right)=h_{\mu}(f) \text {. }
$$

The statements of both of these theorems as given above are actually slightly different from those in the papers referenced, in that we iterate $f$ backwards as well as forwards. We leave these (minor) modifications to the industrious reader. For a complete treatment of entropy see [2].

\section{Lyapunov charts and some estimates}

Let $f: M S$ be a diffeomorphism of a surface and let $\mu$ be an $f$-invariant ergodic Borel probability measure. Then

$$
\frac{1}{n} \log \left\|D f^{n}\right\| \rightarrow \lambda_{1}
$$


and

$$
-\frac{1}{n} \log \left\|D f^{-n}\right\| \rightarrow \lambda_{2}
$$

$\mu$-almost everywhere as $n \rightarrow+\infty$. These numbers are called the $\mu$-Lyapunov exponents of $f$. For more information see [16], [17] or [20].

Let $R(\rho)$ be the square $[-\rho, \rho] \times[-\rho, \rho]$ in $\mathbb{R}^{2}$. If $f$ is $C^{1+\alpha}(\alpha>0)$ and $\varepsilon>0$ is fixed, then at a.e. $x$ one can construct a Lyapunov chart (referred to as the ' $x$-chart' here). These charts are given by

$$
\Phi_{x}: R(A(x)) \rightarrow M
$$

and satisfy

(1) $\Phi_{x} 0=x$,

(2) $A\left(f^{ \pm 1} x\right) \geq(1-\varepsilon) A(x)$

and

(3) if $\tilde{f}_{x}: x$-chart $\rightarrow f x$-chart is the map induced by $f$ and $\chi_{i}=e^{\lambda_{i}}, i=1,2$, then

$$
\left\|\tilde{f}_{x}-\left(\begin{array}{cc}
\chi_{1} & 0 \\
0 & \chi_{2}
\end{array}\right)\right\|_{x_{C 1}}<\varepsilon
$$

Euclidean distance in the $x$-chart is denoted by $\|\cdot\|_{x}$ while $d$ always stands for the Riemannian metric on $M$. These metrics are related by the following inequalities:

$$
d(\cdot, \cdot) \leq K\|\cdot-\cdot\|_{x} \text { for some universal constant } K
$$

and

$$
\|\cdot-\cdot\|_{x} \leq K(x) d(\cdot, \cdot) \text { for some function } K(x) .
$$

For simplicity of notation we will refer to the point $\Phi_{x}^{-1} y$ in the $x$-chart as simply ' $y$ '.

The following estimates are used for the connecting maps between charts.

LemMa 2.2. Suppose $2 \varepsilon<\chi_{1}-1,1-\chi_{2}$ and for $k=1,2, \ldots, g_{k}: \mathbb{R}^{2} \rightarrow \mathbb{R}^{2}$ satisfy $\mathrm{g}_{k} 0=0$ and

$$
\left\|g_{k}-\left(\begin{array}{cc}
\chi_{1} & 0 \\
0 & \chi_{2}
\end{array}\right)\right\|_{C^{1}}<\varepsilon
$$

Then

(1) for any $\rho>0$,

$$
g_{k} \circ \cdots \circ g_{1} R(\rho) \subset R\left(\left(\chi_{1}+2 \varepsilon\right)^{k} \rho\right)
$$

and

(2) if $y_{1}$ and $y_{2}$ denote the first and second coordinates of $y \in \mathbb{R}^{2}$ respectively and $x \in \mathbb{R}^{2}$ is s.t. $\left|x_{1}\right| \geq\left|x_{2}\right|$ then

$$
\left|\left(g_{k} \circ \cdots \circ g_{1} x\right)_{1}\right| \geq\left|\left(g_{k} \circ \cdots \circ g_{1} x\right)_{2}\right|
$$

and

$$
\left|\left(g_{k} \circ \cdots \circ g_{1} x\right)_{1}\right| \geq\left(x_{1}-2 \varepsilon\right)^{k}\left|x_{1}\right|
$$




\section{Proof of the main result}

Let $\mu$ be a Borel probability measure on a compact metric space $X$. Define

$$
\operatorname{HD}(\mu)=\inf _{\substack{Y \subset X \\ \mu Y=1}} \operatorname{HD}(Y) \text {. }
$$

If $X$ is a manifold, $f$ is a $C^{1}$ diffeomorphism of $X$ into itself and $\mu$ is an $f$-invariant ergodic measure, then for every measurable set $Y \subset X$ with $\mu Y>0$, we have $\mu\left(\bigcup_{n \in Z} f^{n} Y\right)=1$ and hence

$$
\operatorname{HD}(Y)=\operatorname{HD}\left(\bigcup_{n \in \mathbf{Z}} f^{n} Y\right) \geq \operatorname{HD}(\mu) .
$$

Thus in this case it is also true that

$$
\operatorname{HD}(\mu)=\inf _{\substack{Y \subset X \\ \mu Y>0}} \operatorname{HD}(Y)
$$

THEOREM 3.1. Let $f: M \circlearrowleft$ be a $C^{1+\alpha}(\alpha>0)$ diffeomorphism of a compact tiwodimensional Riemannian manifold $M$ and let $\mu$ be an f-invariant ergodic Borel probability measure with exponents $\lambda_{1} \geq \lambda_{2}$. Then

$$
\mathrm{HD}(\mu)=h_{\mu}(f)\left[\frac{1}{\lambda_{1}}-\frac{1}{\lambda_{2}}\right]
$$

whenever the right side of this equation $\neq 0 / 0$.

(F. Ledrappier has inequalities along similar lines [12].)

Proof. If $\lambda_{1}$ or $\lambda_{2}=0$, then $h_{\mu}(f)=0$ and there is nothing to prove. If $\lambda_{1}$ and $\lambda_{2}$ are both positive or both negative, then $\mu$ is supported on a finite set [10] and the equality holds trivially. Thus we may assume that $\lambda_{1}>0$ and $\lambda_{2}<0$. We will produce a set $\Lambda \subset M$ such that $\mu \Lambda=1$ and for every measurable set $Y$ with $\mu Y>0$,

$$
\operatorname{HD}(Y \cap \Lambda)=h_{\mu}(f)\left(\frac{1}{\lambda_{1}}-\frac{1}{\lambda_{2}}\right) \text {. }
$$

This follows from proposition 2.1 and the volume lemma below.

Lemma 3.2 (volume lemma). Same setting as in theorem 3.1. Assume also that $\lambda_{1}>0>\lambda_{2}$. Then

for $\mu$-a.e. $x$.

$$
\lim _{\rho \rightarrow 0} \frac{\log \mu B_{\rho}(x)}{\log \rho}=h_{\mu}(f)\left[\frac{1}{\lambda_{1}}-\frac{1}{\lambda_{2}}\right]
$$

We give the proof of this lemma in two parts.

Part I of proof. Let $\Lambda=\{x \in M: x$ is regular in the sense of Oseledec-Pesin and $\left.\lim _{\varepsilon \rightarrow 0} \lim \inf _{n_{1}, n_{2} \rightarrow \infty}-1 /\left(n_{1}+n_{2}\right) \log \mu V\left(x, \varepsilon, n_{1}, n_{2}\right)=h_{\mu}(f)\right\} . \quad\left(V\left(x, \varepsilon, n_{1}, n_{2}\right)\right.$ is defined in $\S 2 \mathrm{~B}$.) As before write $\chi_{i}=e^{\lambda_{1}}, i=1,2$. For every $x \in \Lambda$ and $\varepsilon>0$, we show

$$
\liminf _{\rho \rightarrow 0} \frac{\log \mu B_{\rho}(x)}{\log \rho} \geq\left(h_{\mu}(f)-\varepsilon\right)\left[\frac{1}{\log \frac{\chi_{1}+2 \varepsilon}{1-\varepsilon}}+\frac{1}{\log \frac{\chi_{2}^{-1}+2 \varepsilon}{1-\varepsilon}}\right] \text {. }
$$


Consider Lyapunov charts along the orbit of $x$ as in $\S 2$. All notations are as in that section. Choose $\rho_{0}=\rho_{0}(x)$ s.t.

$$
\liminf _{n_{1}, n_{2} \rightarrow \infty} \frac{-1}{n_{1}+n_{2}} \log \mu V\left(x, \rho_{0}, n_{1}, n_{2}\right) \geq h_{\mu}(f)-\varepsilon
$$

and let $\rho_{1}=\min \left\{A(x), \rho_{0} / K\right\}$. For $\rho \ll \rho_{1}$ define $n_{1}(\rho)$ and $n_{2}(\rho)$ to be the largest integers satisfying

$$
\begin{aligned}
\rho\left(\chi_{1}+2 \varepsilon\right)^{n_{1}(\rho)} & \leq \frac{1}{2}(1-\varepsilon)^{n_{1}(\rho)} \rho_{1}, \\
\rho\left(\chi_{2}^{-1}+2 \varepsilon\right)^{n_{2}(\rho)} & \leq \frac{1}{2}(1-\varepsilon)^{n_{2}(\rho)} \rho_{1} .
\end{aligned}
$$

We claim that for small $\rho$,

$$
B_{K(x)^{-1} \rho}(x) \subset V\left(x, \rho_{0}, n_{1}(\rho), n_{2}(\rho)\right) .
$$

Let $d(x, y) \leq K(x)^{-1} \rho$. Then $y \in R(\rho)$ in the $x$-chart. The connecting maps between charts satisfy the hypotheses of lemma 2.2. Therefore

$$
f^{k} y \in R\left(\left(\chi_{1}+2 \varepsilon\right)^{k} \rho\right)
$$

in the $f^{k} x$-chart for $0 \leq k \leq n_{1}(\rho)$. This makes sense since

$$
A\left(f^{k} x\right) \geq A(x)(1-\varepsilon)^{k} \geq(1-\varepsilon)^{k} \rho_{1} \geq\left(\chi_{1}+2 \varepsilon\right)^{k} \rho .
$$

Back on the manifold, this implies that

$$
d\left(f^{k} x, f^{k} y\right) \leq K 2 \rho\left(\chi_{1}+2 \varepsilon\right)^{k} \leq \rho_{0}
$$

for $0 \leq k \leq n_{1}(\rho)$. A symmetric argument backwards proves the claim.

Now with

$$
n_{1}(\rho) \leq \frac{\log \frac{1}{2} \rho_{1}-\log \rho}{\log \frac{\chi_{1}+2 \varepsilon}{1-\varepsilon}} \leq n_{1}(\rho)+1
$$

and

$$
n_{2}(\rho) \leq \frac{\log \frac{1}{2} \rho_{1}-\log \rho}{\log \frac{\chi^{-1}+2 \varepsilon}{1-\varepsilon}} \leq n_{2}(\rho)+1
$$

we have

$$
\begin{aligned}
\frac{\log \mu B_{K(x)^{-1} \rho}(x)}{\log K(x)^{-1} \rho} \geq & \frac{\log \mu V\left(x, \rho_{0}, n_{1}(\rho), n_{2}(\rho)\right)}{\log \rho-\log K(x)} \\
\geq & {\left[\frac{-1}{n_{1}(\rho)+n_{2}(\rho)+2} \log \mu V\left(x, \rho_{0}, n_{1}(\rho), n_{2}(\rho)\right]\right.} \\
& \cdot\left[\frac{\log \frac{1}{2} \rho_{1}-\log \rho}{\log K(x)-\log \rho}\right]\left[\frac{1}{\log \frac{\chi_{1}+2 \varepsilon}{1-\varepsilon}}+\frac{1}{\log \frac{\chi^{-1}+2 \varepsilon}{1-\varepsilon}}\right] .
\end{aligned}
$$

The desired result follows by taking lim inf. 
Part II of proof. It suffices to show that for every $\varepsilon, \delta>0, \exists \Lambda_{1}=\Lambda(\varepsilon, \delta)$ with $\mu \Lambda_{1}>1-\delta$ s.t. for a.e. $x \in \Lambda_{1}$,

$$
\limsup _{\rho \rightarrow 0} \frac{\log \mu B_{\rho}(x)}{\log \rho} \leq h_{\mu}(f)\left[\frac{1}{\log \left(\chi_{1}-2 \varepsilon\right)}+\frac{1}{\log \left(\chi_{2}^{-1}-2 \varepsilon\right)}\right] .
$$

Many of the ideas in this proof are in [13].

Let $\Lambda_{1}$ be a Pesin set (i.e. a uniformly hyperbolic set) with $\mu \Lambda_{1}>1-\delta$. Then

$$
K_{1}=\sup _{x \in \Lambda_{1}} K(x)<\infty
$$

and

$$
A_{1}=\inf _{x \in \Lambda_{1}} A(x)>0 .
$$

We may assume that $A_{1} K \leq 1$. By Poincaré recurrence we may assume also that every $x \in \Lambda_{1}$ returns to $\Lambda_{1}$ in both forward and backward times. For $x \in \Lambda_{1}$, let

$$
r_{1}(x)=\text { the smallest integer } k>0 \text { s.t. } f^{k} x \in \Lambda_{1}
$$

and

$$
r_{2}(x)=\text { the smallest integer } k>0 \text { s.t. } f^{-k} x \in \Lambda_{1} .
$$

Define a function $\phi: M \rightarrow \mathbb{R}$ by

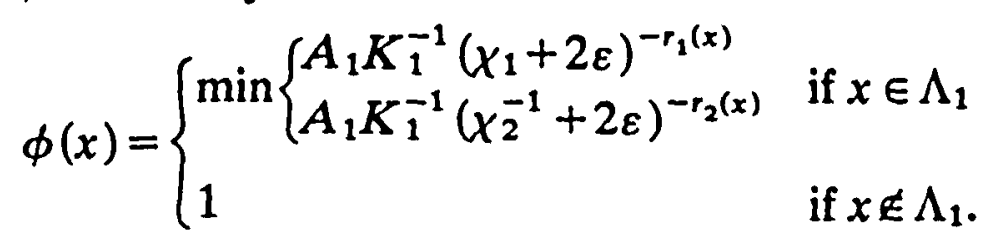

Since

$$
\int_{\Lambda_{1}} r_{1}(x) d \mu=\int_{\Lambda_{1}} r_{2}(x) d \mu=1,
$$

we have $\int-\log \phi<\infty$ and Mañé's estimate

$$
\limsup _{n_{1}, n_{2} \rightarrow \infty} \frac{-1}{n_{1}+n_{2}} \log \mu V\left(x, \phi, n_{1}, n_{2}\right) \leq h_{\mu}(f)
$$

applies to a.e. $x$.

Note that for $x \in \Lambda_{1}$, there are charts at $x, f x, \ldots, f^{r_{1}(x)} x$ of size at least

$$
A_{1}(1-\varepsilon)^{r_{1}(x)}, A_{1}(1-\varepsilon)^{r_{1}(x)-1}, \ldots, A_{1}
$$

respectively and that the connecting maps $\tilde{f}_{f^{k} x}$ satisfy $\tilde{f}_{f^{k}} 0=0$ and

$$
\left\|\tilde{f}_{f^{k} x}-\left(\begin{array}{cc}
\chi_{1} & 0 \\
0 & \chi_{2}
\end{array}\right)\right\|_{C^{1}}<\varepsilon
$$

whenever they are defined. Thus if $x \in \Lambda_{1}$ and $d(x, y) \leq \phi(x)$, then

$$
\|x-y\|_{x} \leq A_{1}\left(\chi_{1}+2 \varepsilon\right)^{-r_{1}(x)}
$$

and by lemma 2.2 ,

$$
\left\|f^{k} x-f^{k} y\right\|_{f_{x}} \leq A\left(f^{k} x\right) \text { for } 0 \leq k \leq r(x) .
$$


For small $\rho>0$, define $n_{1}(\rho)$ and $n_{2}(\rho)$ to be the smallest integers satisfying

$$
\left(\chi_{1}-2 \varepsilon\right)^{n_{1}(\rho)} \rho \geq A_{1}
$$

and

$$
\left(\chi_{2}^{-1}-2 \varepsilon\right)^{n_{2}(\rho)} \rho \geq A_{1} .
$$

We claim that $V\left(x, \phi, n_{1}(\rho), n_{2}(\rho)\right) \subset B_{2 K_{\rho}}(x)$ for all $x \in \Lambda_{1}$. The desired lim sup estimate then works at those points in $\Lambda_{1}$ for which Mané's entropy estimate is valid with respect to the function $\phi$.

It remains to prove the claim. Fix $x \in \Lambda_{1}$ and small $\rho>0$. Let

$$
y \in V\left(x, \phi, n_{1}(\rho), n_{2}(\rho)\right) .
$$

In the $x$-chart, write $y=\left(y_{1}, y_{2}\right)$. Suppose $\left|y_{1}\right| \geq\left|y_{2}\right|$. We prove by looking at forward iterates of $f$ that $\left|y_{1}\right| \leq \rho$. If $\left|y_{2}\right| \geq\left|y_{1}\right|$, a symmetric argument backwards gives $\left|y_{2}\right| \leqslant \rho$.

Let $\left\{s_{0}, s_{1}, \ldots\right\}=\left\{k \geq 0: f^{k} x \in \Lambda_{1}\right\}$. Assume that

$$
0=s_{0}<s_{1}<\cdots<s_{n} \leq n_{1}(\rho)<s_{n+1}<\cdots .
$$

Since $d\left(f^{s} x, f^{s} y\right) \leq \phi\left(f^{s} x\right)$ for $0 \leq i \leq n$, we have, by an earlier argument, $f^{k} y \in$ $f^{k} x$-chart for $0 \leq k \leq n_{1}(\rho)$. The derivatives of the connecting maps between these charts satisfy the hypotheses of lemma 2.2. Thus

$$
\begin{aligned}
\left|\left(f^{s_{n}} y\right)_{1}\right| & \geq\left(\chi_{1}-2 \varepsilon\right)^{s_{n}}\left|y_{1}\right| \\
& \geq \frac{A_{1}}{\left(\chi_{1}-2 \varepsilon\right)^{n_{1}(\rho)-s_{n}}} \frac{\left|y_{1}\right|}{\rho} \\
& \geq \frac{A_{1}}{\left(x_{1}+2 \varepsilon\right)^{r_{1}\left(f^{s_{n x}}\right)}} \frac{\left|y_{1}\right|}{\rho} \\
& \geq \phi\left(f^{\left.s_{n} x\right) K_{1}} \frac{\left|y_{1}\right|}{\rho} .\right.
\end{aligned}
$$

By hypothesis we have $d\left(f^{s_{n}} x, f^{s_{n}} y\right) \leq \phi\left(f^{s_{n}} x\right)$, which implies that

$$
\left|\left(f^{s} n y\right)_{1}\right| \leq \phi\left(f^{s} x\right) K_{1}
$$

in the $f^{s} x$-chart. This proves $\left|y_{1}\right| / \rho \leq 1$.

Note. It should be pointed out that part I of this proof could be done without Lyapunov charts. The estimates there are in fact valid for $C^{1}$ diffeomorphisms.

\section{Other notions of fractional dimension}

Closely related to Hausdorf dimension is capacity, introduced by Kolmogorov. If a probability measure is present, Rényi, using the information function, defined yet another notion of dimension. For arbitrary sets and arbitrary measures the differences between these definitions are real: examples can easily be constructed where they differ sharply. In the context of our main theorem, however, these notions all turn out to be the same. 


\section{A. Capacity}

Let $X$ be a compact metric space. For $\varepsilon>0$ let $N(\varepsilon)$ be the minimum number of $\varepsilon$-balls needed to cover $X$. Define the lower capacity of $X$,

$$
C(X)=\liminf _{\varepsilon \rightarrow 0} \frac{\log N(\varepsilon)}{\log (1 / \varepsilon)}
$$

and the upper capacity or simply capacity of $X$,

$$
\vec{C}(X)=\limsup _{\varepsilon \rightarrow 0} \frac{\log N(\varepsilon)}{\log (1 / \varepsilon)^{\circ}}
$$

If the dimension of a set is to be related to the $\mu$-entropy and $\mu$-exponents of a map, that notion of dimension must be sensitive to the 'good points' of $\mu$. Capacity, however, does not distinguish between a set and its closure. Ledrappier has made the following modification to correct this insensitivity [12]: Let $\mu$ be a Borel probability measure on $X$. For $\varepsilon, \delta>0$, let $N(\varepsilon, \delta)$ be the minimum number of $\varepsilon$-balls needed to cover a set of $\mu$-measure $\geq 1-\delta$. Then

$$
C_{\mathrm{L}}(\mu) \stackrel{\text { def }}{=} \sup _{\delta \rightarrow 0} \liminf \frac{\log N(\varepsilon, \delta)}{\log (1 / \varepsilon)}
$$

and

$$
\bar{C}_{L}(\mu) \stackrel{\text { def }}{=} \sup _{\delta \rightarrow 0} \limsup \frac{\log N(\varepsilon, \delta)}{\log (1 / \varepsilon)} .
$$

Another (technically different) modification of capacity that is perhaps more in line with our definition of $\mathrm{HD}(\mu)$ is:

$$
\begin{aligned}
& C(\mu) \stackrel{\text { def }}{=} \sup _{\delta \rightarrow 0} \inf _{\substack{Y \subset X \\
\mu Y \geq 1-\delta}} C(Y), \\
& \bar{C}(\mu) \stackrel{\text { def }}{=} \sup _{\delta \rightarrow 0} \inf _{\substack{Y \subset X \\
\mu Y \geq 1-\delta}} \bar{C}(Y) .
\end{aligned}
$$

It is clear that

$$
\underline{C}_{\mathrm{L}}(\mu) \leq \underline{C}(\mu) \text { and } \bar{C}_{\mathrm{L}}(\mu) \leq \bar{C}(\mu) .
$$

Proposition 4.1. Let $\mu$ be a Borel probability measure on a compact metric space $X$. Then HD $(\mu) \leq C_{L}(\mu)$.

Proof. Fix any $C>C_{L}(\mu)$ and $\alpha>0$. For $n=1,2, \ldots$ let $\delta_{n}=\alpha / 2^{n}$. Choose $\varepsilon_{n} \downarrow 0$ and sets $A_{n}$ with $\mu A_{n} \geq 1-\delta_{n}$ s.t. $A_{n}$ is covered by $N_{n}$ balls of radius $\varepsilon_{n}$ and

$$
\frac{\log N_{n}}{\log 1 / \varepsilon_{n}} \leq C \text {. }
$$

Let $A=\bigcap_{n=1} A_{n \cdot}$. Then $\mu A \geq 1-\alpha$ and for each $\varepsilon_{n,}$

$$
\inf _{\substack{x \rightarrow \text { nver of } A \\ \text { diem } x \leq s_{n}}} \sum_{B \in \times}(\operatorname{diam} B)^{C} \leq N_{n} \varepsilon_{n}^{C} \leq 1 .
$$

Thus $\operatorname{HD}(A) \leq C$ and hence $\exists Y \subset X$ with $\mu Y=1$ s.t. $H D(Y) \leq C$.

The next proposition is the capacity analogue of proposition 2.1 . 
Proposition 4.2. Let $\mu$ be a continuous Borel probability measure on $\mathbb{R}^{n}$ and let $\Lambda \subset \mathbb{R}^{n}$ be measurable and bounded. Suppose that $\mu \Lambda>0$. Suppose also that for every $x \in \Lambda$,

$$
\underline{\delta} \leq \liminf _{\rho \rightarrow 0} \frac{\log \mu B_{\rho}(x)}{\log \rho} \leq \limsup \frac{\log \mu B_{\rho}(x)}{\log \rho} \leq \bar{\delta} .
$$

Then

$$
\delta \leq C(\mu) \leq \bar{C}(\mu) \leq \bar{\delta} .
$$

Proof. It follows from propositions 2.1 and 4.1 that

$$
C(\mu) \geq \underline{C}_{L}(\mu) \geq \mathrm{HD}(\mu) \geq \underline{\delta} .
$$

To show $\bar{C}(\mu) \leq \bar{\delta}$, it suffices to prove that for $\eta>\bar{\delta}$ and $\delta>0, \exists U \subset \Lambda$ with $\mu U>\mu \Lambda-\delta$ s.t. $\bar{C}(U) \leq \eta$. For any set $U$, let

$$
\mathscr{P}(U, \varepsilon)=\left\{B_{\varepsilon}(x): x \in U\right\}
$$

and define

$$
\beta(U, \mu)=\inf \left\{\beta: \lim _{\varepsilon \rightarrow 0} \inf _{\substack{\alpha=\text { cover of } U \\ \kappa \subset \mathscr{P}(U, \varepsilon)}} \sum_{B \in \kappa}(\mu B)^{\beta}=0\right\} .
$$

The rest of the proof goes as in proposition 2.1.

These results are summarized in theorem 4.4 at the end of this section.

\section{B. Rényi dimension}

Let $\mu$ be a Borel probability measure on a compact metric space $X$. If $\kappa$ is a finite partition of $X$, let

$$
H_{\mu}(\kappa)=-\sum_{A \in \kappa} \mu A \log \mu A
$$

and let

$$
H_{\mu}(\varepsilon)=\inf _{\substack{\kappa \\ \text { diam } \kappa \leq \varepsilon}} H_{\mu}(\kappa) .
$$

We can think of $H_{\mu}(\varepsilon)$ as the minimum amount of information any $\varepsilon$-partition gives. The Rényi dimension of the pair $(X, \mu)$ measures the growth of this information relative to $\varepsilon$. We write

$$
\underline{R}(\mu)=\liminf _{\varepsilon \rightarrow 0} \frac{H_{\mu}(\varepsilon)}{\log 1 / \varepsilon}
$$

and

$$
\bar{R}(\mu)=\limsup _{\varepsilon \rightarrow 0} \frac{H_{\mu}(\varepsilon)}{\log 1 / \varepsilon} .
$$

Rényi's original definition is in [18].

Proposinon 4.3. Let $\mu$ be a Borel probability measure on a compact metric space $X$ with $\bar{C}(X)<\infty$. Then

(1) $\bar{R}(\mu) \leq \bar{C}_{L}(\mu)$ 
and

(2) if for $\mu$-a.e. $x$,

$$
\liminf _{\rho \rightarrow 0} \frac{\log \mu B_{\rho}(x)}{\log \rho} \geq \alpha_{0}
$$

then

$$
\underline{R}(\mu) \geq \alpha_{0}
$$

Proof. (1) Fix small $\delta>0$. Let $B_{1}, \ldots, B_{N(\varepsilon, \delta)}$ be $\varepsilon$-balls that together cover a set of measure $\geq 1-\delta$. Let $\tilde{B}_{1}=B_{1}$ and

$$
\tilde{B}_{n}=B_{n}-\bigcup_{i=1}^{n-1} \tilde{B}_{i} \quad \text { for } n=2,3, \ldots, N(\varepsilon, \delta)
$$

Let $U_{1}, \ldots, U_{N(\varepsilon)}$ be $\varepsilon$-balls that cover all of $X$ and let

$$
\tilde{U}_{1}=U_{1}-U \tilde{B}_{i}, \tilde{U}_{n}=U_{n}-\bigcup_{i=1}^{n-1} \tilde{U}_{i}-U \tilde{B}_{i}
$$

Then $\kappa=\left\{\tilde{B}_{1}, \ldots, \tilde{B}_{N(\varepsilon, \delta)}, \tilde{U}_{1}, \ldots, \tilde{U}_{N(\varepsilon)}\right\}$ is a partition of $X$ with diam $\kappa \leq 2 \varepsilon$. Using the fact that if $\sum_{i=1}^{s} p_{i}=t, 0 \leq p_{i} \leq 1$ and $0<t \leq 1$ then

$$
-\sum_{i=1}^{s} p_{i} \log p_{i} \leq-t \log t+t \log s
$$

we have

$$
\begin{aligned}
H_{\mu}(2 \varepsilon) & \leq H_{\mu}(\kappa) \\
& \leq \log N(\varepsilon, \delta)+[-\delta \log \delta+\delta \log N(\varepsilon)]
\end{aligned}
$$

Thus

$$
\begin{aligned}
\bar{R}(\mu) & =\limsup _{\varepsilon \rightarrow 0} \frac{H_{\mu}(2 \varepsilon)}{\log 1 / 2 \varepsilon} \\
& \leq \limsup _{\varepsilon \rightarrow 0} \frac{\log N(\varepsilon, \delta)}{\log 1 / 2 \varepsilon}+\limsup _{\varepsilon \rightarrow 0} \frac{[-\delta \log \delta+\delta \log N(\varepsilon)]}{\log 1 / 2 \varepsilon} \\
& \leq \bar{C}_{L}(\mu)+\delta \bar{C}(X) .
\end{aligned}
$$

Let $\delta \rightarrow 0$.

(2) Let $\alpha<\alpha_{0}$ and $\delta>0$ be given. There exists $U \subset M$ with $\mu U>1-\delta$ and $\rho_{0}>0$ s.t. $\forall \rho \leq \rho_{0}$ and $\forall x \in U$,

$$
\mu B_{\rho}(x) \leq \rho^{\alpha}
$$

Let $\kappa=\left\{A_{1}, \ldots, A_{r}\right\}$ be any finite partition with diam $\kappa \leq \varepsilon \leq \rho_{0}$. It suffices to show that

$$
\frac{H_{\mu}(\kappa)}{\log 1 / \varepsilon} \geq(1-2 \delta) \alpha
$$


Let $\beta_{1}=\left\{A_{i} \in \kappa: A_{i} \cap U \neq \varnothing\right\}$ and $\beta_{2}=\kappa-\beta_{1}$. Then $\mu\left(\cup \beta_{2}\right)<\delta$. Each $A_{i} \in \beta_{1}$ is contained in $B_{\varepsilon}\left(x_{l}\right)$ for some $x_{l} \in U$ and therefore has $\mu$-measure $\leq \varepsilon^{\alpha}$. Thus

$$
\begin{aligned}
H_{\mu}(\kappa) & \geq-\sum_{A_{i} \in \beta_{1}} \mu A_{i} \log \mu A_{i} \\
& \geq \frac{1-2 \delta}{\varepsilon^{\alpha}}\left(-\varepsilon^{\alpha} \log \varepsilon^{\alpha}\right) \\
& =(1-2 \delta) \alpha \log \frac{1}{\varepsilon} .
\end{aligned}
$$

This proves the claim.

Propositions 2.1, 4.1, 4.2 and 4.3 together prove the following result:

THEOREM 4.4. Let $\mu$ be a Borel probability measure on a compact Riemannian manifold and suppose for $\mu$-a.e. $x$,

$$
\lim _{\rho \rightarrow 0} \frac{\log \mu B_{\rho}(x)}{\log \rho}=\alpha_{0}
$$

Then

$$
\mathrm{HD}(\mu)=C(\mu)=\bar{C}(\mu)=\underline{C}_{L}(\mu)=\bar{C}_{L}(\mu)=\underline{R}(\mu)=\bar{R}(\mu)=\alpha_{0} .
$$

It is probably fair to call this number $\operatorname{dim}(\mu)$ if it exists. In particular we have ${ }^{*}$

Corollary 4.5. Let $f: M \circlearrowleft$ be a $C^{1+\alpha}$ diffeomorphism of a compact Riemannian 2-manifold and let $\mu$ be an f-invariant ergodic Borel probability measure with non-zero exponents $\lambda_{1} \geq \lambda_{2}$. Then

$$
\operatorname{dim}(\mu)=h_{\mu}(f)\left[\frac{1}{\lambda_{1}}-\frac{1}{\lambda_{2}}\right]
$$

\section{Corollaries and remarks}

A. Higher dimensions

If $\operatorname{dim} M \geq 3$, there can be no simple formula that works in all cases. (See [22: § 5].)

COROLlary 5.1. Let $f: M \unlhd$ be a $C^{1+\alpha}$ diffeomorphism of a compact Riemannian manifold $M$ and let $\mu$ be an ergodic Borel probability measure with non-zero $f$ exponents $\lambda_{1} \geq \cdots \geq \lambda_{r}$. Let

$$
\lambda_{u}=\min \left\{\lambda_{1}: \lambda_{l}>0\right\}
$$

Then

$$
h_{\mu}(f)\left[\frac{1}{\lambda_{1}}-\frac{1}{\lambda_{r}}\right] \leq \mathrm{HD}(\mu) \leq h_{\mu}(f)\left[\frac{1}{\lambda_{u}}-\frac{1}{\lambda_{u+1}}\right] .
$$

The proof is the same as that of Theorem 3.1. We do not know whether better estimates of this type can be made or whether generically some equality might hold.

B. Bifurcations of horseshoes

We first remark that if $f: M^{2} \leftrightarrows$ is Axiom $A$ [21], then our estimates work in the $C^{1}$ category. Consider a continuous one-parameter family $\left\{f_{t}\right\}_{t \in I}$ of Axiom A 
diffeomorphisms on a surface. Let $\Lambda_{t}$ be a basic set or horseshoe of $f_{t}$ and assume that the topological structure of $f_{t} \mid \Lambda_{t}$ remains unchanged as $t$ varies through $I$. We state some results on the 'size' of $\Lambda_{t}$ with respect to some of the interesting measures.

Corollary 5.2. Let $\left\{f_{t}\right\}$ and $\left\{\Lambda_{t}\right\}$ be as described above. If

(1) $\mu_{t}$ is the measure maximizing entropy for $f_{t} \mid \Lambda_{t}$ or

(2) $f_{t}$ is $C^{1+\alpha}, \Lambda_{t}$ is an attractor and $\mu_{t}$ is the Bowen-Ruelle measure [4], then $\operatorname{dim}\left(\mu_{t}\right)$ varies continuously with $t$.

Proof. For (1), $h_{\mu_{t}}\left(f_{t}\right)$ remains constant while

$$
\lambda_{1}(t)=\int \log \left|D f_{t}^{u}\right| d \mu_{t} \quad \text { and } \quad \lambda_{2}(t)=\int \log \left|D f_{t}^{s}\right| d \mu_{t}
$$

vary continuously with $t$. The claim follows from theorem 3.1. For (2), first observe that if $(\Lambda, f)$ is one fixed basic set and $\phi_{t}: \Lambda \rightarrow \mathbb{R}$ is a continuous one-parameter family of Hölder continuous functions, then the equilibrium state of $\left(f, \phi_{t}\right)$ varies continuously. (See [3] and [4].) Here $h_{\mu_{1}}\left(f_{t}\right)=\lambda_{1}(t)$ and $\lambda_{1}(t)$ and $\lambda_{2}(t)$ vary continuously as before.

C. The case $\operatorname{dim}(\mu)=2$

In the setting of theorem 3.1, $\operatorname{dim}(\mu)=2$ if and only if $\lambda_{1}=-\lambda_{2}=h_{\mu}(f)$. This happens only in very special circumstances for Axiom A diffeomorphisms. (See [4].)

COROLlary 5.3. If $f: M^{2} \subseteq$ is a $C^{1+\alpha}$ Axiom $A$ diffeomorphism and $\mu$ is an ergodic Borel probability measure, then $\operatorname{dim}(\mu)=2$ iff $M^{2}$ is a torus, $f$ is Anosov and $\mu$ is equivalent to Riemannian measure.

We do not know whether HD $(\mu)=$ topological dimension of the ambient manifold implies that $\mu$ is smooth for $C^{2}$ diffeomorphisms in general.

D. Lyapunov dimension and Yorke's conjectures

If $f: \Lambda \unlhd$ is an attractor and $\lambda_{1} \geq \cdots \geq \lambda_{r}$ are Lyapunov exponents of $f$ with respect to a certain measure, Yorke defines the Lyapunov dimension of this attractor (with respect to this measure) to be

$$
k+\frac{\lambda_{1}+\cdots+\lambda_{k}}{\left|\lambda_{k+1}\right|}
$$

where $k=\max \left\{i: \lambda_{1}+\cdots+\lambda_{i}>0\right\}$. He conjectures ([8], [9]) that for 'most' maps, Lyapunov dimension $\leq$ capacity and that. under some additional hypotheses it is equal to Rényi dimension.

We verify at least this much:

COROLlary 5.4. For diffeomorphisms of surfaces, if $h_{\mu}(f)=\lambda_{1}$, then $\mu$-Lyapunov dimension $=\operatorname{dim}(\mu)$.

The hypothesis that $h_{\mu}(f)=\lambda_{1}$ is satisfied in the case of Axiom A attractors with $\mu$ being the Bowen-Ruelle measure. Conceivably many other attractors may adritit such an invariant measure. 
Ledrappier has suggested reformulating Yorke's conjecture in variational form. He has proved [12: prop. 3] that if $f: M S$ is a diffeomorphism and $\mu$ is an ergodic Borel probability measure, then .

$$
\bar{C}_{L}(\mu)-\mu \text {-Lyap } \operatorname{dim} \leq 0 \text {. }
$$

Corollary 5.4 then gives 'equilibrium states' for this variational equation in the case of surfaces.

This research was partially supported by NSF Grant MCS- 8002781 .

\section{REFERENCES}

[1] A. S. Besicovitch. Sets of fractional dimension II: On the um of digits of real numbers represented in the dyadic system. Math. Ann. 110 (1934), 321-329.

[2] P. Billingsley. Ergodic Theory and Information. Wiley: New York, 1965.

[3] R. Bowen. Some systems with unique equilibrium states. Math. Systems Theory. 8 (1974), 193-202.

[4] R. Bowen. Equilibrium States and the Ergodic Theory of Anosov Diffeomorphisms. Lecture Notes in Math. no. 470. Springer: Berlin, 1975.

[5] M. Brin \& A. Katok. On local entropy. Preprint.

[6] A. Douady \& J. Oesterlé. Dimension de Hausdorff des attracteurs. C.R. Acad. Sci. 24 (1980), $1135-1138$.

[7] H. G. Eggleston. The fractional dimension of a set defined by decimal properties. Quart. J. Math. Oxford Ser. 20 (1949), 31-36.

[8] P. Frederickson, J. Kaplan, E. Yorke \& J. Yorke. The Liapunov dimension of strange attractors. Preprint.

[9] J. Kaplan \& J. Yorke. Chaotic Behavior of Multidimensional Difference Equations. Lecture Notes in Math. no. 730, pp. 228-237. Springer: Berlin, 1979.

[10] A. Katok. Lyapunov exponents, entropy and periodic orbits for diffeomorphisms. Publ. Math. I.H.E.S. 51 (1980), 137-174.

[11] A. N. Kolmogorov. A new invariant for transitive dynamical systems. Dokl. Akad. Nauk SSSR. 119 (1958), 861-864.

[12] F. Ledrappier. Some relations between dimension and Lyapunov exponents. Commun. Math. Phys. 81 (1981), 229-238.

[13] R. Mañé. A proof of Pesin's formula. Ergod. Th. \& Dynam. Sys. 1 (1981), 95-102.

[14] A. Manning. A relation between Lyapunov exponents, Hausdorff dimension and entropy. Ergod. Th. \& Dynam. Sys. 1 (1981), 451-459.

[15] Z. Nitecki. Differentiable Dynamics. MIT Press: Cambridge, Mass., 1971.

[16] V. I. Oseledec. A multiplicative ergodic theorem, Lyapunov characteristic numbers for dynamical systems. Trudy Moskov. Mat. Obšč. 19 (1968), 179-210, Engl. translation.

[17] Ja. Pesin. Characteristic Lyapunov exponents and smooth ergodic theory. Russ. Math. Surveys. 32 (1977), 55-114.

[18] A. Rényi. Dimension, entropy and information. Transactions of the second Prague Conference on Information Theory, Statistical Decision Functions, Random Processes (1957) 545-556.

[19] D. Ruelle. An inequality for the entropy of differentiable maps. Bol. Soc. Bras. Mat. 9 (1978), 83-87.

[20] D. Ruelle. Ergodic theory of differentiable dynamical systems. Publ. Mfath. I.H.E.S. 50 (1979), 27-58.

[21] S. Smale. Differentiable dynamical systems. Bull. Amer. Math. Soc. 73 (1967), 747-817.

[22] L. Young. Capacity of astractors. Ergod. Th. \& Dynam. Sys. 1 (1981), 381-388. 